\title{
Prevalence of central obesity and its associated risk factors among adults in
}

\section{Southeast Ethiopia: a community-based cross-sectional study}

Yohannes Tekalegn ${ }^{*}$, Damtew Solomon ${ }^{2}$, Biniyam Sahiledengle ${ }^{1}$, Tesfaye Assefa ${ }^{3}$, 4 Wegene Negash ${ }^{3}$, Anwar Tahir ${ }^{3}$, Tadele Regassa ${ }^{2}$, Ayele $\mathrm{Mamo}^{4}$, Habtamu Gezahegn², 5 Kebebe Bekele ${ }^{5}$, Demisu Zenbaba ${ }^{1}$, Alelign Tasew ${ }^{1}$, Fikreab Desta ${ }^{1}$, Daniel Atilaw ${ }^{2}$, 6 Zegeye Regassa ${ }^{3}$, Fikadu Nugusu, Zinash Teferu Engida ${ }^{1}$, Degefa Gomora Tesfaye ${ }^{6}$, Chala 7 Kene $^{6}$, Wondu Shiferaw Nigussie ${ }^{3}$, Dereje Chala ${ }^{3}$, Adisu Gemechu Abdi ${ }^{3}$, Girma Beressa, ${ }^{1}$

8 Demelash Woldeyohannes ${ }^{7}$, Heather L. Rogers ${ }^{8}$, Lillian Mwanri ${ }^{9}$

10 'Public Health Department, Goba Referral Hospital, Madda Walabu University, Bale Goba, Ethiopia

11 2Biomedical Department, Goba Referral Hospital, Madda Walabu University, Ethiopia

12 3Nursing Department, Goba Referral Hospital, Madda Walabu University, Bale Goba, Ethiopia

13 4Pharmacy Department, Goba Referral Hospital, Madda Walabu University, Bale Goba, Ethiopia

14 5Surgery Department, Goba Referral Hospital, Madda Walabu University, Bale Goba, Ethiopia

$15{ }^{6}$ Midwifery Department, Goba Referral Hospital, Madda Walabu University, Bale Goba, Ethiopia

$16{ }^{7}$ Wachemo University, School of Medicine and Health Science, Department of Public health, Hosana,

17 Ethiopia

$18{ }^{8}$ Biocruces Bizkaia Health Research Institute, Barakaldo, Bizkaia, Spain 48903 and Ikerbasque Basque

19 Foundation for Science Bilbao, Bizkaia, Spain 48009.

$20{ }^{9}$ Torrens University Australia, 88 Wakefield St, Adelaide SA 5000.

*Corresponding author: [Yohannes Tekalegn]

23 Email: yohannesefa@gmail.com

24 Tel: +251913578901 


\section{Abstract}

27 Background: Obesity and overweight are known are public health scourge challenges affecting

28 populations across the world. These conditions have been associated with a wide range of chronic diseases including type 2 diabetes mellitus, cardiovascular disease, and cancers. In Ethiopia, literature regarding the burden of central (abdominal) obesity is scarce. This study aimed to fill this gap by assessing the prevalence and risk factors associated with central obesity among adults in Ethiopia.

33 Methods: From May to July 2021, a community-based cross-sectional survey was conducted on

34 a sample of 694 adults aged $\geq 18$ years in administrative towns of Bale zone, Southeast Ethiopia.

35 Multi-stage sampling followed by systematic random sampling was employed to identify study participants. Waist and hip circumferences were measured using standard protocols. The World Health Organization STEPS wise tool was used to assess risk factors associated with central obesity. Bi-variable and multi-variable binary logistic regression were used to identify factors associated with central obesity. Adjusted odds ratios (AOR) and their corresponding 95\% confidence intervals (CI) were reported to estimate the strength of associations.

Results: The overall prevalence of central obesity using waist circumference was $39.01 \%$ (15.44\% for men and $53.12 \%$ for women). Multi-variable binary logistic regression analysis revealed that female sex $(A O R=12.93,95 \% \mathrm{CI}$ : 6.74-24.79), Age groups: $30-39$ years old $(\mathrm{AOR}=2.8,95 \% \mathrm{CI}$ : 1.59-4.94), 40-49 years (AOR=7.66, 95\% CI: 3.87-15.15), 50-59 years (AOR=4.65, 95\% CI: 2.199.89), $\geq 60$ years $(\mathrm{AOR}=12.67,95 \% \mathrm{CI}: 5.46-29.39)$, occupational status like: housewives $(\mathrm{AOR}=5.21,95 \% \mathrm{CI}: 1.85-14.62)$, self-employed workers $(\mathrm{AOR}=4.63,95 \% \mathrm{CI}: 1.62-13.24)$, government/private/non-government employees ( $\mathrm{AOR}=4.68,95 \% \mathrm{CI}: 1.47-14.88)$ and skipping breakfast ( $\mathrm{AOR}=0.46,95 \% \mathrm{CI}$ : 0.23-0.9) were significantly associated with central obesity.

49 Conclusions: Abdominal obesity has become an epidemic in towns of southeast Ethiopia, and the

50 prevalence is higher among women. Female sex, older age group, being employed, not skipping 51 breakfast were significantly associated with central obesity.

52 Keywords: Central obesity, abdominal obesity, obesity, prevalence, risk factors, Ethiopia 


\section{Introduction}

56 The World Health Organization (WHO) defines obesity as abnormal or excess fat accumulation

57 that may impair health (1). Obesity has been associated with the imbalance between intake and energy expenditure, a condition that may be caused by either non-modifiable or modifiable factors (2). This caloric imbalance creates an excess accumulation of energy which in turn is stored in the

60 body resulting in excess body weight. Although genetic factors are not modifiable, obesity can

61 result from a complex interaction between environmental, socio-economic, and/or personal

62 behaviors, factors that are modifiable. Addressing the modifiable factors play as one of the critical

63 strategies in preventing obesity (3). Globally, the prevalence of overweight and obesity increased

64 by $27.5 \%$ for adults and $47.1 \%$ for children between 1980 and 2013 . The number of individuals

65 with overweight and obesity increased from 857 million in 1980 to 2.1 billion in 2013 (4). If this

66 secular trend continues persistently, $38 \%$ of the world's adult population will be overweight and

67 another $20 \%$ will be obese by 2030 (5).

68 Worldwide, a high body mass index (BMI) has been reported to be responsible for 4 million deaths

69 and 120 million disability-adjusted life years (6). Several studies have further documented obesity

70 as the risk factor for many non-communicable diseases (NCDs) and chronic health conditions

71 including hypertension, high lipid concentrations, type 2 diabetes, coronary heart disease, stroke,

72 and certain cancers (7-22). Central (abdominal) obesity, measured in waist circumference, waist

73 to hip ratio, and waist to height ratios, is highly linked with increased risk of morbidity and

74 mortality and is considered to be superior to BMI in predicting cardiovascular disease and

75 mortality risks (23-25).

76 Many low and middle-income countries (LMICs), including Ethiopia, currently face a double

77 burden of malnutrition (26). While LMICs are dealing with problems of infectious diseases and

78 undernutrition, they are also experiencing a rapid increase in non-communicable disease risk

79 factors like overweight and obesity (1, 27-29), and the emergence of Corona Virus Disease

80 (COVID-19) pandemic has further challenged health systems, economies and populations across

81 the globe, but and more severely in LMICs $(30,31)$. Evidence suggests that NCDs are dramatically

82 increasing in Ethiopia and it was estimated that NCDs were responsible for 711 deaths per 100,000

83 population in 2015 (32). Cardiovascular diseases, cancer, diabetes, and mental disorders were 
84 responsible for $30 \%$ of the total disease burden in Ethiopia as measured in age-standardized 85 disability-adjusted life years (DALYs) rates in 2017 (33).

86 In Ethiopia, epidemiological studies regarding the prevalence, distribution, and determinants of 87 obesity are meager. More specifically, few studies have assessed the prevalence and risk factors 88 of central (abdominal) obesity (34-39), a superior predictor of NCDs. The prevalence reported in 89 the aforementioned previous studies ranges from $15.5 \%$ in the northern part of Ethiopia (36) to $9037.4 \%$ in southwest Ethiopia (39). Similarly, risk factors identified by those studies vary from 91 district to district which warrants studying the prevalence and context-based risk factors in our 92 study setting which has implications for prevention efforts / public health campaigns. To the best 93 of our knowledge, there is no published evidence on the magnitude and factors associated with 94 central obesity in Southeast Ethiopia. The current study aims to assess the prevalence of central 95 (abdominal) obesity and its associated risk factors in the administrative towns of Bale Zone, 96 Southeast Ethiopia.

\section{Methods and Materials}

\section{Study design, setting and subjects}

99 From May to July 2021, a community-based cross-sectional study was conducted to assess the 100 prevalence of central (abdominal) obesity among adults ( $\geq 18$ years) in the administrative towns of 101 Bale zone, Southeast Ethiopia. All adults residing in the study area for at least 6 months were 102 eligible for inclusion. However, potential participants were excluded if they had: psychiatric 103 problems, hearing impairments, body deformities (kyphosis and scoliosis), other debilitations and/ 104 or handicaps. Pregnant women were also ineligible for inclusion. Bale zone, one of the Regional 105 States in Ethiopia in the Southeastern part of Oromia (an area of 43,690.56 $\mathrm{km}^{2}$ ), is located 106 between $5^{0} 22^{\prime}-8^{0} 08^{\prime}$ latitude north and between $38^{0} 41^{\prime}-40^{0} 44^{\prime}$ longitude east. (Figure 1). 107 According to the Central Statistical Agency (CSA), in 2007 (latest available report), the Bale zone, 108 had a total population of 1,402,492, including 713,517 men and 688,975 women, an increase of $10915.16 \%$ from the 1994 Census. Of the total inhabitants in Bale Zone, 166,758 (26.20\% were) urban 110 dwellers and 44,610 (3.18\% were) pastoralists. The zone comprises 18 districts and a total of 111297,081 (an average of 4.72 persons per household) households. There are two administrative 112 towns in the zone namely Robe and Goba towns. Furthermore, each administrative town is divided 
113 into smaller administrative clusters known as 'gots'. Robe and Goba towns have 36 and 24 gots

114 respectively. According to the 2021 administrative report, Robe and Goba towns respectively had 115 population of 73,152 and 52,785 people.

\section{Sample size determination and sampling procedures}

117 Based on the total population in the zone, the study sample size of 700 individuals was calculated 118 using OpenEpi (Version 3, an open-source calculator)considering the following parameters: 95\% 119 level of confidence, $4 \%$ margin of error, $24.4 \%$ reported prevalence of abdominal obesity by the 120 previous study in Dilla town, South Ethiopia (38), design effect of 2, and non-response rate of $1215 \%$.. A multi-stage stratified sampling and, systematic random sampling were employed to select 122 the study participants for inclusion in the study. Initially, the study populations sampling was 123 stratified into the two (Robe and Goba) towns, followed by stratification into randomly selected 124 clusters (gots). One third of clusters were selected from each town, and proportionate to their 125 number, eight and 12 gots were selected from Goba and Robe respectively. Furthermore, 126 households in the sampled clusters were selected using systematic sampling techniques and one 127 adult per sampled household was selected using the lottery method.

\section{Data collection and measurement procedures}

129 Interviewer-administered, structured questionnaires were used to collect data on socio130 demographic and behavioural characteristics followed by physical measurements of weight, 131 height, waist, and hip circumferences. A standard questionnaire was adapted from the WHO 132 STEPS-wise questionnaire for chronic disease risk factor surveillance (40). The English version 133 of the questionnaire was translated to the local languages spoken in the study area, Afan Oromo 134 and Amharic. After the translation into local languages, the questionnaire was back-translated to 135 English to check the consistency. Weight was measured using an electronic digital weight scale 136 by putting the scale on a firm flat surface after participants took off footwear, heavy clothes, and 137 empty their pockets for heavy items. Participants' height measurements were taken in a standing 138 position by a portable height measuring board placed on a firm surface against a wall. With 139 participants facing the data collector, feet placed together, and eyes leveled at the ears, readings 140 were taken in centimeters (to the nearest millimeter). With the participants' arms relaxed at the 141 sides, the waist circumferences were measured by constant tension tape at the end of a normal 
142 expiration; at the midpoint between the lower margin of the last palpable rib and the top of the 143 iliac crest (hip bone). Waist measurements read at the level of the tape to the nearest millimeter, 144 while ensuring the tape was comfortably tight enough not to cause compression of the skin. Hip 145 circumferences were measured by constant tension tape with the arms relaxed at the sides at the 146 maximum circumference over the buttocks. Hip circumferences were measured and read at the 147 level of the tape to the nearest millimeter. Further details on the study physical measurement 148 protocols are consistent with the WHO STEPS-wise instrument guideline (41).

149 Data collection was conducted by six data collectors (three male-female pairs) with degrees of 150 bachelor in health sciences (Nursing, Public Health, and Midwifery) Female and male data 151 collectors respectively collected female and male participants' data. Two supervisors with a degree 152 master of public health took part in overseeing the data collection process. Data collectors and 153 supervisors were provided with a two-day intensive training on the objective of the study, 154 administration protocols for the questionnaires, administration protocols for anthropometric 155 measurements (weight, height, waist, and hip circumferences), and how to maintain confidentiality 156 and privacy of the study participants. With assistance from the study supervisors, all questionnaires 157 were checked every day by data collectors for completeness before leaving the data collection site 158 (household).

\section{Outcome variable}

160 Central (abdominal) obesity is the dependent variable for this study, which is derived from waist 161 circumference measurement. As per World Health Organization recommendations, waist162 circumference $>94$ centimeters for males and $>80$ centimeters for females were categorized as 163 central obesity (23). Individuals with central obesity were coded as " 1 " and others were coded as 164 "0".

\section{Independent variables}

166 Independent variables included sociodemographic and behavioral variables. Sociodemographic 167 variables included the town of residence (Robe or Goba); age (categorized into 18-29, 30-39, 40$16849,50-59$, and $\geq 60$ years); sex (male or female), marital status (categorized as never married, $169 \mathrm{married} / \mathrm{cohabiting}$, or divorced/separated/widowed); educational status (categorized as no formal 170 education, secondary education, or diploma and above); occupational status (housewives, self- 
171 employed, government/non-government/private employee, student/unemployed, or Retired);

172 family size (categorized as $\leq 2,>2$ ); and wealth index (computed by principal component analysis

173 using household assets and rank-ordered as low, medium or high wealth index terciles). Behavioral

174 variables included characteristics such as fruit and vegetable consumption (categorized as less than

175 five servings of fruit and/or vegetables per day, or five or more servings of fruit and/or vegetables

176 per day), skipping breakfast (categorized as yes or no), avoidance of eating foods prepared outside

177 the home (categorized as yes or no) and levels of physical activities [derived by calculating the

178 metabolic equivalent value (MET) - study participants with an equivalent combination of moderate

179 and vigorous-intensity physical activity achieving at least 600 MET-minutes were categorized as

180 sufficient level while the others were categorized as insufficient physical activity levels], sedentary

181 activities were measure by adding total time spent sitting or reclining on a typical day, current

182 smokers of tobacco were categorized as yes or no and participants who consumed alcohol were

183 categorized as never drank, consumed in last 12 months, or consumed in last 30 days.

184 Measurements including fruit and/or vegetable consumption, level of physical activities, alcohol,

185 and/or tobacco consumption were assessed and analyzed using World Health Organization (WHO)

186 STEPS Surveillance tool recommendations (41).

\section{Data analysis procedures}

188 The data was coded and entered into EpiData Version 3.1 and cleaned, processed, and analyzed 189 using SPSS version 25 and STATA version 14. The variables were described using mean, 190 frequencies, proportions, and tables. The Chi-square test was used to check the statistical 191 difference of the distribution of categorical independent variables between men and women. A 192 two-sample Wilcoxon rank-sum (Mann-Whitney) test was used to check the statistical difference 193 in the distribution of continuous independent variables between men and women. Both bi-variable 194 and multi-variable binary logistic regression analyses were used to identify factors associated with 195 the outcome variable. Variables having a p-value of less than 0.25 in the bi-variable binary logistic 196 regression model were included in the multivariable binary logistic regression analysis model. 197 After checking for multi-collinearity, enter method was used to run the model. The logit of the 198 dependent variable was checked for outlier and 7 outlying values (having standardized residual $199>2.58$ at the level of $\alpha<0.01$ ) were excluded from the analysis. Hosmer and Lemeshow's goodness 200 of model fit was checked and the data fitted the model well $(\mathrm{p}=0.92)$. Multi-collinearity between 
201 independent variables was checked using variance inflation factor (VIF), the mean VIF was 2.1

202 which is less than the recommended cut-off values (42). Finally, adjusted odds ratios with 95\%

203 confidence intervals were used to estimate the strength of associations between the outcome

204 variable and independent variables. All tests were two-tailed and statistical significances were

205 declared at p-value $<0.05$.

206 Ethical considerations

207 Ethical clearance and support letters to introduce the researchers and the study to respective study 208 areas were obtained from the ethical review committee of Madda Walabu University. Permission 209 letters to conduct the survey were obtained from the respective authorities of the two towns (Goba 210 and Robe). The methods were conducted following the tenets of the Helsinki declaration. To obtain 211 oral informed consent an information sheet was read to all eligible study participants before data 212 were collected. The privacy of the respondents was respected and data were de-identified before 213 analysis and were reported in aggregate.

\section{Results}

\section{Sociodemographic characteristics of study participants}

216 A total of 694 adults (259 men and 435 women) participated in this study, a response rate of $99.1 \%$.

217 Four hundred and eight (58.8\%) and 286 (41.2\%) study participants were from Robe and Goba 218 town respectively. Participants' age ranged from 18-95 years old. Men participants' median age 219 was 41 years old with an interquartile range (IQR) of 28-55 years. women's median age was 32 220 years with an IQR of 25-45 years (Table 1).

221 Table 1: Sociodemographic characteristics of study participants in administrative towns of Bale 222 Zone, Southeast Ethiopia, 2021.

\begin{tabular}{|l|l|l|l|l|l|}
\hline Variables & $\begin{array}{l}\text { Total (\%), } \\
\mathbf{N = 6 9 4}\end{array}$ & $\begin{array}{l}\text { Men (\%), } \\
\mathbf{n = 2 5 9}\end{array}$ & $\begin{array}{l}\text { Women } \\
\mathbf{( \% )} \\
\mathbf{n}=\mathbf{4 3 5}\end{array}$ & $\begin{array}{l}\text { Pearson } \\
\text { chi } \\
\text { square } \\
(\mathbf{d f})\end{array}$ & p-value \\
\hline Town of residence & & & & & \\
\hline Robe & $408(58.8)$ & $161(62.2)$ & $247(56.8)$ & $\begin{array}{l}29.2(4) \\
(1)\end{array}$ & $<0.001^{*}$ \\
\hline Goba & $286(41.2)$ & $98(37.8)$ & $188(43.2)$ & & \\
\hline
\end{tabular}




\begin{tabular}{|c|c|c|c|c|c|}
\hline \multicolumn{6}{|l|}{ Age category } \\
\hline $18-29$ years & $262(37.8)$ & $79(30.6)$ & $183(42.2)$ & $29.2(4)$ & $<0.001 *$ \\
\hline $30-39$ years & $134(19.4)$ & $41(15.9)$ & $93(21.4)$ & & \\
\hline $40-49$ years & $103(14.9)$ & $37(14.3)$ & $66(15.2)$ & & \\
\hline $50-59$ years & $92(13.3)$ & $52(20.2)$ & $40(9.2)$ & & \\
\hline$\geq 60$ years & $101(14.6)$ & $49(19.0)$ & $52(12.0)$ & & \\
\hline \multicolumn{6}{|l|}{ Educational status } \\
\hline No formal education & $98(14.1)$ & $23(8.9)$ & $75(17.2)$ & $33.7(3)$ & $<0.001^{*}$ \\
\hline Primary education $(1-8)$ & $205(29.6)$ & $54(20.9)$ & $151(34.7)$ & & \\
\hline Secondary education (9-12) & $219(31.6)$ & $94(36.4)$ & $\begin{array}{l}125 \\
(28.74) \\
\end{array}$ & & \\
\hline Diploma and above & $171(24.7)$ & $87(33.7)$ & $84(19.3)$ & & \\
\hline \multicolumn{6}{|l|}{ Ethnicity } \\
\hline Oromo & $546(78.7)$ & $216(83.4)$ & $330(75.9)$ & $4.8(3)$ & 0.1 \\
\hline Amhara & $114(16.4)$ & $33(12.8)$ & $81(18.6)$ & & \\
\hline Wolaita & $11(1.6)$ & $4(1.5)$ & $7(1.6)$ & & \\
\hline Others ${ }^{\mathbf{a}}$ & $13(1.9)$ & $4(1.5)$ & $9(2.0)$ & & \\
\hline Refused & $10(1.4)$ & $2(0.8)$ & $8(1.8)$ & & \\
\hline \multicolumn{6}{|l|}{ Religion } \\
\hline Muslim & $291(41.9)$ & $116(44.8)$ & $175(40.2)$ & $2.79(3)$ & 0.4 \\
\hline Orthodox Christian & $333(48.0)$ & $119(46.0)$ & $214(49.2)$ & & \\
\hline Protestant Christian & $69(9.9)$ & $23(8.9)$ & $46(10.6)$ & & \\
\hline Catholic & $1(0.1)$ & $1(0.4)$ & $0(0.0)$ & & \\
\hline \multicolumn{6}{|l|}{ Marital status } \\
\hline Never married & $139(20.0)$ & $68(26.3)$ & $71(16.3)$ & $27.4(2)$ & $<0.001^{*}$ \\
\hline Married/cohabiting & $465(67.0)$ & $177(68.3)$ & $288(66.2)$ & & \\
\hline Divorced/separated/widowed & $90(13.0)$ & $14(5.4)$ & $76(17.5)$ & & \\
\hline \multicolumn{6}{|l|}{ Occupational status } \\
\hline Housewives & $239(34.8)$ & - & $239(55.2)$ & $\begin{array}{l}219.1 \\
(4)\end{array}$ & $<0.001^{*}$ \\
\hline Self-employed & $195(28.4)$ & $112(44.1)$ & $83(19.2)$ & & \\
\hline $\begin{array}{l}\text { Government/private/NGO } \\
\text { employed }\end{array}$ & $113(16.5)$ & $69(27.2)$ & $44(10.2)$ & & \\
\hline Retired & $38(5.5)$ & $25(9.8)$ & $13(3.0)$ & & \\
\hline Student/unemployed & $102(14.8)$ & $48(18.9)$ & $54(12.5)$ & & \\
\hline \multicolumn{6}{|l|}{ Family size } \\
\hline$\leq 2$ & $391(56.3)$ & $121(46.7)$ & $270(62.1)$ & $15.5(1)$ & $<0.001^{*}$ \\
\hline$>2$ & $303(43.7)$ & $138(53.3)$ & $165(37.9)$ & & \\
\hline \multicolumn{6}{|l|}{ Wealth index } \\
\hline Low & $279(40.2)$ & $85(32.8)$ & $194(44.6)$ & $10.5(2)$ & $0.005^{*}$ \\
\hline Medium & $161(23.2)$ & $63(24.3)$ & $98(22.5)$ & & \\
\hline High & $254(36.6)$ & $111(42.9)$ & $143(32.9)$ & & \\
\hline
\end{tabular}

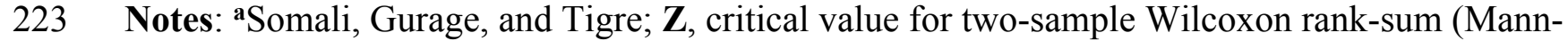

224 Whitney) test; * significant at $\mathrm{p}<0.05$ 
medRxiv preprint doi: https://doi.org/10.1101/2022.02.24.22271452; this version posted February 25, 2022. The copyright holder for this preprint (which was not certified by peer review) is the author/funder, who has granted medRxiv a license to display the preprint in perpetuity.

It is made available under a CC-BY 4.0 International license.

\section{Behavioral characteristics of the study participants}

227 More than half (58\%) of the study participants consumed less than five servings of fruit and/or

228 vegetable per day. The mean number of days of fruit were consumed per week was 2.3 days.

229 Approximately $15 \%$ of the study participants reported skipping breakfast and another 15\% avoided

230 eating foods prepared outside the home. Nearly $29 \%$ of the study participants had an insufficient

231 level of physical activity as per World Health Organization recommendations (Table 2).

232 Table 2: Behavioral characteristics of study participants in administrative towns of Bale zone,

233 Southeast Ethiopia, 2021.

\begin{tabular}{|l|l|l|l|l|l|}
\hline Variables & $\begin{array}{l}\text { Total (\%), } \\
\mathbf{N = 6 9 4}\end{array}$ & $\begin{array}{l}\text { Men (\%), } \\
\mathbf{n = 2 5 9}\end{array}$ & $\begin{array}{l}\text { Women } \\
\mathbf{( \% )} \\
\mathbf{n}=\mathbf{4 3 5}\end{array}$ & $\begin{array}{l}\text { Pearson } \\
\text { chi } \\
\text { square } \\
\text { (df) }\end{array}$ & p-value \\
\hline $\begin{array}{l}\text { Number of days eat fruit } \\
\text { and/or vegetables on } \\
\text { average per week }\end{array}$ & & & & & \\
\hline No fruit and/or vegetable & $58(8.4)$ & 30 & $28(6.4)$ & $77(3)$ & 0.05 \\
\hline $1-2$ & $349(50.4)$ & 118 & $231(53.1)$ & & \\
\hline $3-4$ & $219(31.6)$ & 87 & $132(30.3)$ & & \\
\hline$>=5$ & $67(9.7)$ & 23 & $44(10.1)$ & & \\
\hline $\begin{array}{l}\text { Number of servings of } \\
\text { fruit and/or vegetables on } \\
\text { average per day }\end{array}$ & & & & & \\
\hline $1-2$ servings & & & & & \\
\hline $3-4$ servings & $49(8.1)$ & $17(8.0)$ & $32(8.2)$ & $0.4(2)$ & 0.8 \\
\hline$>=5$ servings & $301(49.8)$ & $102(48.3)$ & $199(50.9)$ & & \\
\hline $\begin{array}{l}\text { Avoid eating foods } \\
\text { prepared outside of a } \\
\text { home }\end{array}$ & $252(41.7)$ & $92(43.6)$ & $160(40.9)$ & & \\
\hline Yes & & & & & \\
\hline No & & & & & \\
\hline Skip breakfast & $584(84.6)$ & $176(68.2)$ & $408(94.4)$ & $85.4(1)$ & $<0.001^{*}$ \\
\hline No & $106(15.4)$ & $82(31.8)$ & $24(5.6)$ & & \\
\hline Yes & $592(14.5)$ & $210(81.4)$ & $382(88.0)$ & $5.7(1)$ & $0.01 *$ \\
\hline Level of physical activity & $100(88.5)$ & $48(18.6)$ & $52(12.0)$ & & \\
\hline $\begin{array}{l}\text { Insufficient physical } \\
\text { activity }\end{array}$ & $195(28.8)$ & $31(12.3)$ & $164(38.6)$ & $53.3(1)$ & $<0.01^{*}$ \\
\hline Sufficient physical activity & $482(71.2)$ & $221(87.7)$ & $261(61.4)$ & & \\
\hline
\end{tabular}




\begin{tabular}{|l|l|l|l|l|l|}
\hline $\begin{array}{l}\text { Number of days fruit } \\
\text { consumed in a typical } \\
\text { week (mean } \pm \text { SD) }\end{array}$ & $2.33 \pm 1.60$ & $\begin{array}{l}2.31 \pm \\
1.57\end{array}$ & $\begin{array}{l}2.35 \pm \\
1.62\end{array}$ & $\mathrm{Z}=0.1$ & 0.9 \\
\hline $\begin{array}{l}\text { Number of days } \\
\text { vegetables consumed in a } \\
\text { typical week ( mean } \pm \\
\text { SD) }\end{array}$ & $3.59 \pm 1.97$ & $\begin{array}{l}3.49 \pm \\
1.83\end{array}$ & $\begin{array}{l}3.66 \pm \\
2.05\end{array}$ & $\mathrm{Z}=-0.45$ & 0.6 \\
\hline $\begin{array}{l}\text { Number of servings of } \\
\text { fruit on average per day } \\
\text { ( mean } \pm \text { SD) }\end{array}$ & $2.11 \pm 0.86$ & $\begin{array}{l}2.25 \pm \\
0.99\end{array}$ & $\begin{array}{l}2.04 \pm \\
0.77\end{array}$ & $\mathrm{Z}=2.28$ & $0.02^{*}$ \\
\hline $\begin{array}{l}\text { Number of servings of } \\
\text { vegetables on average per } \\
\text { day (mean } \pm \text { SD) }\end{array}$ & $2.11 \pm 0.96$ & $\begin{array}{l}2.11 \pm \\
0.98\end{array}$ & $\begin{array}{l}2.12 \pm \\
0.95\end{array}$ & $\mathrm{Z}=-0.28$ & 0.7 \\
\hline $\begin{array}{l}\text { Number of servings of } \\
\text { fruit and/or vegetables on } \\
\text { average per day ( mean } \pm \\
\text { SD) }\end{array}$ & $4.2 \pm 1.40$ & $4.36 \pm$ & $4.14 \pm 1.31$ & $\mathrm{Z}=1.35$ & 0.1 \\
\hline $\begin{array}{l}\text { Minutes spent on } \\
\text { sedentary activities on } \\
\text { average per day ( mean } \pm \\
\text { SD) }\end{array}$ & $178.1 \quad 1 \pm$ & $166.65 \pm$ & $184.90 \pm$ & $\mathrm{Z}=-1.78$ & 0.07 \\
\hline
\end{tabular}

Notes: Z, critical value for two-sample Wilcoxon rank-sum (Mann-Whitney) test; ${ }^{*}$ significant at $236 \mathrm{p}<0.05$ consumed alcohol in the past twelve months (Table 3).

241 Table 3: Alcohol and tobacco consumption behavior of study participants in administrative 242 towns of Bale zone, Southeast Ethiopia, 2021.

\begin{tabular}{|l|l|l|l|l|l|}
\hline Variables & $\begin{array}{l}\text { Total } \\
\mathbf{( \% ) ,} \\
\mathbf{N = 6 9 4}\end{array}$ & $\begin{array}{l}\text { Men (\%), } \\
\mathbf{n = 2 5 9}\end{array}$ & $\begin{array}{l}\text { Women } \\
\mathbf{( \% ) ,} \\
\mathbf{n = 4 3 5}\end{array}$ & $\begin{array}{l}\text { Pearson } \\
\text { chi } \\
\text { square } \\
\text { (df) }\end{array}$ & p-value \\
\hline $\begin{array}{l}\text { Currently smoke tobacco } \\
\text { products, } \mathbf{n = 6 9 4}\end{array}$ & & & & & \\
\hline Yes & $7(1.0)$ & $7(2.7)$ & $0(0.0)$ & $\begin{array}{l}11.87 \\
(1)\end{array}$ & $<0.001^{*}$ \\
\hline No & $\begin{array}{l}687 \\
(99.0)\end{array}$ & $\begin{array}{l}252 \\
(97.3)\end{array}$ & $435(100)$ & & \\
\hline Ever consumed alcohol, $\mathbf{n}=\mathbf{6 9 3}$ & & & & & \\
\hline
\end{tabular}




\begin{tabular}{|c|c|c|c|c|c|}
\hline Yes & $\begin{array}{l}222 \\
(32.0)\end{array}$ & $94(36.4)$ & $128(29.4)$ & $3.65(1)$ & 0.05 \\
\hline No & $\begin{array}{l}471 \\
(68.0)\end{array}$ & $\begin{array}{l}164 \\
(63.6)\end{array}$ & $307(70.6)$ & & \\
\hline \multicolumn{6}{|l|}{$\begin{array}{l}\text { Consumed alcohol in past } 12 \\
\text { months, } \mathrm{n}=222\end{array}$} \\
\hline Yes & $\begin{array}{l}212 \\
(95.5)\end{array}$ & $90(94.7)$ & $122(96.1)$ & $0.22(1)$ & 0.6 \\
\hline No & $10(4.5)$ & $5(5.3)$ & $5(3.9)$ & & \\
\hline \multicolumn{6}{|l|}{$\begin{array}{l}\text { Frequency of alcohol drinking in } \\
\text { past } 12 \text { months, } n=222\end{array}$} \\
\hline 5-6 days per week & $1(0.5)$ & $0(0.0)$ & $1(0.8)$ & $\begin{array}{l}38.50 \\
(3)\end{array}$ & $<0.001^{*}$ \\
\hline 1-4 days per week & $25(11.7)$ & $18(20.0)$ & $7(5.7)$ & & \\
\hline 1-3 days per week & $89(41.8)$ & $52(57.8)$ & $37(30.1)$ & & \\
\hline Less than once a month & $97(46.0)$ & $20(22.2)$ & $78(63.4)$ & & \\
\hline \multicolumn{6}{|l|}{$\begin{array}{l}\text { Consumed alcohol within past } 30 \\
\text { days, } n=222\end{array}$} \\
\hline Yes & $\begin{array}{l}134 \\
(60.5)\end{array}$ & $68(71.6)$ & $67(52.3)$ & $8.44(1)$ & $0.004 *$ \\
\hline no & $88(39.5)$ & $27(28.4)$ & $61(47.7)$ & & \\
\hline \multicolumn{6}{|l|}{$\begin{array}{l}\text { Five or more drinks on a single } \\
\text { occasion at least once during the } \\
\text { past } 30 \text { days, } n=135\end{array}$} \\
\hline Yes & $23(17.0)$ & $15(5.8)$ & $8(1.8)$ & $7.91(1)$ & $0.005 *$ \\
\hline No & $\begin{array}{l}112 \\
(83.0)\end{array}$ & $\begin{array}{l}244 \\
(94.2)\end{array}$ & $427(98.2)$ & & \\
\hline $\begin{array}{l}\text { Number of drinking occasions in } \\
\text { past } 30 \text { days among current }(30 \\
\text { days) drinkers ( mean } \pm \text { SD), } \\
n=134\end{array}$ & $\begin{array}{l}2.53 \pm \\
1.96\end{array}$ & $\begin{array}{l}3.13 \pm \\
2.14\end{array}$ & $1.92 \pm 1.54$ & $Z=4.63$ & $<0.001^{*}$ \\
\hline $\begin{array}{l}\text { Number of standard drinks per } \\
\text { drinking occasion among } \\
\text { current (past } 30 \text { days) drinkers } \\
(\text { mean } \pm \text { SD), } n=133\end{array}$ & $\begin{array}{l}2.35 \pm \\
1.20\end{array}$ & $\begin{array}{l}2.73 \pm \\
1.29\end{array}$ & $1.97 \pm 0.98$ & $Z=3.77$ & $<0.001^{*}$ \\
\hline $\begin{array}{l}\text { Maximum number of standard } \\
\text { drinks consumed on one } \\
\text { occasion in the past } 30 \text { days } \\
(\text { mean } \pm \text { SD }), n=134)\end{array}$ & $\begin{array}{l}2.87 \pm \\
.47\end{array}$ & $\begin{array}{l}3.52 \pm \\
1.63\end{array}$ & $2.24 \pm 0.96$ & $Z=5.33$ & $<0.001^{*}$ \\
\hline
\end{tabular}

243 Notes: Z, critical value for two-sample Wilcoxon rank-sum (Mann-Whitney) test; *significant at $244 \mathrm{p}<0.05$ 


\section{Prevalence of central obesity}

247 As described in Table 4, of the 259 men included in this study, 40 (15.44\%, 95\% CI: 11.26-

$24820.43 \%$ ) had waist circumference $>94$ centimeters. Of the 433 women included in the study, 230

$249(53.12 \%, 95 \%$ CI: 48.29-57.89\%) had a waist circumference greater than 80 centimeters. Based 250 on the waist-to-hip ratios, 204 (47.11\%, 95\% CI: 42.32, 51.93\%) and 129 (49.80\%, 95\% CI: 43.55-

$25156.06 \%$ ) of women and men had wait to hip ratio of greater than or equal to 0.85 and 0.90 , 252 respectively.

253 Table 4: Distribution of central obesity by sex among adult populations in administrative towns 254 of Bale zone, Southeast Ethiopia, 2021.

\begin{tabular}{|l|l|l|}
\hline Central obesity distribution by sex & Frequency & Percent (95\% CI) \\
\hline Men (n= 259) & & \\
\hline Waist circumference $>94$ centimeters & 40 & $15.44(11.26-20.43)$ \\
\hline Waist circumference $>102$ centimeters & 9 & $3.47(1.60-6.49)$ \\
\hline Waist to hip ratio $\geq 0.9$ & 129 & $49.81(43.55-56.06)$ \\
\hline Women (n=433) & & \\
\hline Waist circumference $>80$ centimeters & 230 & $53.12(48.29-57.89)$ \\
\hline Waist circumference $>88$ centimeters & 118 & $27.19(23.11-31.70)$ \\
\hline Waist to hip ratio $\geq 0.85$ & 204 & $47.11(42.32-51.93)$ \\
\hline a Overall (both sexes) $\mathbf{N}=\mathbf{6 9 2}$ & & \\
\hline Centrally obese & 270 & $39.01(35.36-42.76)$ \\
\hline
\end{tabular}

Notes: ${ }^{\text {a }}$ calculated using waist circumference $>94$ and $>80$ centimeters for men and women respectively.

\section{Factors associated with central (abdominal) obesity}

258 Findings of the multivariable binary logistic regression analysis revealed that women had higher

259 odds of central obesity compared to men (AOR:12.93, 95\% CI: 6.74-24.79). Age groups 30-39 260 (AOR: 2.80, 95\% CI: 1.59-4.94), 40-49 (AOR: 7.66, 95\% CI: 3.87-15.15), 50-59 (AOR: 4.65, 95\%

261 CI: 2.19-9.89), and $\geq 60$ years (AOR: 12.67 95\% CI: 5.46-29.39) had higher odds of central obesity 262 compared to those below 30 years old. Likewise, Government/Non-governmental/Private 263 employees (AOR: 4.68, 95\% CI: 1.47-14.88), self-employed (AOR: 4.63, 95\% CI: 1.62-13.24), 264 and housewives (AOR: 5.21,95\% CI: 1.85-14.62) were more likely to be centrally obese compared 265 to unemployed or students. Moreover, those individuals who skip breakfast were a 54\% reduced 266 risk of central obesity compared to their counterparts (Table 5). 
medRxiv preprint doi: https://doi.org/10.1101/2022.02.24.22271452; this version posted February 25, 2022. The copyright holder for this preprint (which was not certified by peer review) is the author/funder, who has granted medRxiv a license to display the preprint in perpetuity.

It is made available under a CC-BY 4.0 International license .

267 Table 5: Factors associated with central obesity among adult men in administrative towns of Bale 268 zone, Southeast Ethiopia, 2021.

\begin{tabular}{|c|c|c|c|c|}
\hline \multirow[t]{3}{*}{ Variables } & \multicolumn{2}{|c|}{ Abdominal obesity } & \multirow[t]{3}{*}{ COR (95\% CI) } & \multirow[t]{3}{*}{$\operatorname{AOR}^{a}(95 \%$ CI $)$} \\
\hline & Yes & No & & \\
\hline & n (\%) & n (\%) & & \\
\hline \multicolumn{5}{|l|}{ Residential town } \\
\hline Goba & $124(43.4)$ & $162(56.6)$ & $1.4(1.0-1.8)$ & $1.10(0.72-1.70)$ \\
\hline Robe & $146(36.0)$ & $260(64.0)$ & 1 & 1 \\
\hline \multicolumn{5}{|l|}{ Sex } \\
\hline Male & $40(15.4)$ & $219(84.6)$ & 1 & 1 \\
\hline Female & $230(53.1)$ & $203(46.9)$ & $6.2(4.2-9.1)^{*}$ & $12.93(6.74-24.79)^{* *}$ \\
\hline \multicolumn{5}{|l|}{ Age in years } \\
\hline $18-29$ & $56(21.4)$ & $206(78.6)$ & 1 & 1 \\
\hline $30-39$ & $59(44.4)$ & $74(55.6)$ & $2.9(1.9-4.6)^{*}$ & $2.80(1.59-4.94)^{* *}$ \\
\hline $40-49$ & $61(59.2)$ & $42(40.8)$ & $5.3(3.3-8.7)^{*}$ & $7.66(3.87-15.15)^{* *}$ \\
\hline $50-59$ & $37(40.2)$ & $55(59.8)$ & $2.5(1.5-4.1)^{*}$ & $4.65(2.19-9.89)^{* *}$ \\
\hline$>=60$ & $56(56.0)$ & $44(44.0)$ & $4.7(2.9-7.7)^{*}$ & $12.67(5.46-29.39)^{* *}$ \\
\hline \multicolumn{5}{|l|}{ Marital status } \\
\hline Never married & $19(13.7)$ & $120(86.3)$ & 1 & 1 \\
\hline Married/cohabiting & $195(42.1)$ & $268(57.9)$ & $4.6(2.7-7.7)^{*}$ & $1.38(0.63-3.05)$ \\
\hline Divorced/separated/widowed & $56(62.2)$ & $34(37.8)$ & $10.4(5.5-19.8)^{*}$ & $1.05(0.40-2.78)$ \\
\hline \multicolumn{5}{|l|}{ Educational status } \\
\hline No formal education & $62(63.3)$ & $36(36.7)$ & $4.9(2.9-8.5)^{*}$ & $1.66(0.70-3.90)$ \\
\hline Primary education (1-8) & $100(49.3)$ & $103(50.7)$ & $2.8(1.8-4.3)^{*}$ & $1.38(0.68-2.80)$ \\
\hline Secondary education (9-12) & $64(29.2)$ & $155(70.8)$ & $1.2(0.8-1.9)$ & $0.94(0.48-1.86)$ \\
\hline Diploma and above & $44(25.7)$ & $127(74.3)$ & 1 & 1 \\
\hline \multicolumn{5}{|l|}{ Occupational status } \\
\hline House wives & $154(61.1)$ & $98(38.9)$ & $11.7(6.0-22.4)^{*}$ & $5.21(1.85-14.62)^{* *}$ \\
\hline Self employed & $57(31.0)$ & $127(69.0)$ & $3.3(1.7-6.6)^{*}$ & $4.63(1.62-13.24)^{* *}$ \\
\hline $\begin{array}{l}\text { Government/private/NGO } \\
\text { employed }\end{array}$ & $33(29.2)$ & $80(70.8)$ & $3.1(1.5-6.3)^{*}$ & $4.68(1.47-14.88)^{* *}$ \\
\hline Retired & $12(33.3)$ & $24(66.7)$ & $3.7(1.5-9.3)^{*}$ & $2.78(0.73-10.48)$ \\
\hline Student/unemployed & $12(11.9)$ & $89(88.1)$ & 1 & 1 \\
\hline \multicolumn{5}{|l|}{ Family size } \\
\hline$\leq 2$ & $28(40.0)$ & $42(60.0)$ & 1 & - \\
\hline$>2$ & $184(37.9)$ & $301(62.1)$ & $0.9(0.5-1.5)$ & - \\
\hline \multicolumn{5}{|l|}{ Wealth index } \\
\hline Low & $105(41.3)$ & $173(58.7)$ & 1 & 1 \\
\hline Medium & $60(37.5)$ & $100(62.5)$ & $0.9(0.8-1.6)$ & $0.69(0.40-1.17)$ \\
\hline High & $105(41.3)$ & $149(58.7)$ & $1.2(0.8-1.6)$ & $0.74(0.45-1.22)$ \\
\hline $\begin{array}{l}\text { Fruit and } / \text { or vegetable } \\
\text { consumption on average per } \\
\text { day }\end{array}$ & & & & \\
\hline
\end{tabular}




\begin{tabular}{|l|l|l|l|l|}
\hline Less than five servings per day & $128(36.4)$ & $224(63.6)$ & $0.8(0.5-1.1)$ & $0.87(0.57-1.32)$ \\
\hline Five or more servings per day & $108(43.0)$ & $143(57.0)$ & $\mathbf{1}$ & $\mathbf{1}$ \\
\hline $\begin{array}{l}\text { Avoid eating foods prepared } \\
\text { outside of a home }\end{array}$ & & & & \\
\hline Yes & $245(42.1)$ & $337(57.9)$ & 1 & $\mathbf{1}$ \\
\hline No & $21(19.8)$ & $85(80.2)$ & $0.3(0.2-0.6)^{*}$ & $1.59(0.79-3.18)$ \\
\hline Skip breakfast & & & & \\
\hline No & $242(41.0)$ & $348(59.0)$ & $\mathbf{1}$ & $\mathbf{1}$ \\
\hline Yes & $27(27.0)$ & $73(73.0)$ & $0.1(0.3-0.9)^{*}$ & $0.46(0.23-0.90)^{* *}$ \\
\hline Level of physical activity & & & & \\
\hline Insufficient physical activity & $96(49.5)$ & $98(50.5)$ & $1.9(1.3-2.6)^{*}$ & $0.84(0.55-1.30)$ \\
\hline Sufficient physical activity & $166(34.5)$ & $315(65.5)$ & $\mathbf{1}$ & $\mathbf{1}$ \\
\hline
\end{tabular}

Notes: n, frequency; COR, crude odds ratio; AOR, adjusted odds ratio; CI, confidence interval; aThe model adjusted for: residence, sex, age, marital status, education status, occupation, fruit and vegetable consumption, avoiding eating foods prepared outside the home, skipping breakfast and level of physical activity; *significant at $\mathrm{p}<0.05$ (crude); **significant at $\mathrm{p}<0.05$ (adjusted).

\section{Discussion}

274 The findings of this study reveal that the overall prevalence of central (abdominal) obesity was $39.0 \%$. In comparison to men, women had a higher prevalence of central obesity $(53.1 \%$ vs $15.4 \%$ ). The figures reported in this study are comparable with studies conducted in Gondar and Dabat towns, Northwest Ethiopia (37.6\% \& 33.6\%) (37, 43), but higher than the studies conducted in Nekemte town, West Ethiopia (28.4\%) (35), Woldia town, Northeast Ethiopia (15.5\%) (36), and Dilla town South Ethiopia (24.4\%) (38). The possible variations of the obesity prevalence could be explained by the use of different cutoff values for waist circumferences (36) and the variation of age distributions between the studies participants $(35,38)$. Furthermore, variation in the gender make-up of the samples might be a possible reason for the differences, given such large differences

283 in prevalence found in this study. Though there are slight variations in the prevalence rates across

284 the regions, the magnitude of central obesity in the study appeared to be high. A recent systematic review and meta-analysis in Ethiopia reported that the prevalence of overweight and obesity is 286 increasing especially in urban settings (44). The results of the present study confirm these findings 287 and suggest that gender is a particularly important factor to examine when estimating overall 288 prevalence rates in Ethiopian regions.

289 In this study, women had 13 times higher odds of central obesity compared to men. This finding 290 is corroborated by similar studies in different parts of Ethiopia (35-39). Similarly, studies 
conducted by Jaacks et al., suggested that in countries with stage 1 obesity transition, the prevalence of obesity is higher among women compared to men (45). According to a study conducted by the Global Burden of Diseases report, the trend of obesity and overweight is persistently higher among women than men in developing countries (4). Further appropriately powered longitudinal studies within males and females in Ethiopia examining predictors of obesity/central obesity are warranted.

This study revealed that the odds of central obesity tend to increase as age increases. A longitudinal study conducted by Baum \& Ruhm reported that body weight increases as age increases (46). The positive association between age and abdominal obesity is supported by several other studies conducted elsewhere including in Ethiopia, China, Russian Federation (35-37, 39, 47-50). This association might be explained partly by the gradual decline in physiologic activities and basal metabolic rate as age increases (51).

Employed adults and housewives had higher odds of central obesity compared to unemployed or students. This might be explained by the hypothesis that employed adults might have better access to foods than their counterparts. Previous studies in Ethiopia suggested that unemployed men are less likely to be obese compared to employed men (52). This finding contradicts a study conducted in North Glasgow, UK, which reported that unemployed men and women were less likely to be centrally obese compared to full-time workers (53). This contradiction needs to be addressed by conducting strong epidemiological studies exploring the role of employment status on the development of abdominal obesity in low-income countries. With regarding to findings of the

311 higher odds of central obesity among housewives, it is plausible to hypothesis that staying at home 312 exposes women to ease reach of food. As has stated elsewhere, frequency of meal intakes has 313 been associated with an increase in total energy intake (54), hence increased opportunities to 314 developing obesity.

315 Individuals who reported skipping their breakfast were 54\% less likely to be centrally obese 316 compared to their counterparts. This might be partly explained by the fact that frequency of meals 317 is associated with an increase in total energy intake (54). However, this finding disagreed with a 318 systematic review and meta-analysis conducted by $M a$ et al., which reported skipping breakfast 319 results in a 31\% increase in abdominal obesity (55). In this study, breakfast skipping behavior was 320 measured through self-report and the reason for skipping breakfast was not collected. Moreover, 
321 meal frequency and portion size were not assessed, which could be a source of variation with

322 existing evidence. Furthermore, this study was not topic-specific for the association between

323 skipping breakfast and the risk of central obesity.

324 This study aimed to examine predictors of central (abdominal) obesity in a region with no prior

325 evidence on the distribution and associated risk factors. Although the study used primary data on

326 physical measurements like waist and hip circumferences and the WHO-STEPS wise tool for non-

327 communicable disease risk factors surveillance with trained data collectors and supervisors, the

328 findings must be interpreted in light of the following limitations. First, due to the cross-sectional

329 nature of the study, a cause-effect relationship cannot be established between the risk factors and

330 obesity. Secondly, the study sample was too small for sex and age-specific reporting of the

331 prevalence and risk factors. Differences in prevalence and risk factors by gender suggest the need

332 for gender-specific stratification in larger, appropriately powered longitudinal studies. Lastly, data

333 regarding dietary habits, physical activity, alcohol, and tobacco use were collected through self-

334 reported behavior questionnaires, which might be affected by the recall and social desirability bias.

335 Future studies could use more objective measurements, including third part reporting and 336 wearables to assess these behaviors.

\section{Conclusions}

338 The burden of abdominal obesity is high in southeast Ethiopia, especially in women, with one out 339 of every two women and three out of every 10 men found to be centrally (abdominally) obese.

340 Prevention strategies that account for identified modifiable risk factors are needed to curb the

341 increasing burden of central obesity and its public health impact. Further studies of gender-specific 342 risk factors are warranted.

\section{Acknowledgments}

344 We thank Madda Walabu University for the financial support to conduct this study. We are also 345 grateful to all data collectors, Goba and Robe Health Office and Health extension workers, kebele 346 and Got administrators for their facilitation and cooperation to smoothly conduct this study. 


\section{References}

348 1. World Health Organization. Obesity and overweight 2021 [updated 9 June 2021.

349 Available from: https://www.who.int/news-room/fact-sheets/detail/obesity-and-overweight.

350 2. Kelishadi R, Hashemi Pour M, Sarraf-Zadegan N, Sadry GH, Ansari R, Alikhassy H, et

351 al. Obesity and associated modifiable environmental factors in Iranian adolescents: Isfahan Healthy Heart Program - heart health promotion from childhood. Pediatrics international. 2003;45(4):435-42.

3543 . Hruby A, Hu FB. The Epidemiology of Obesity: A Big Picture. PharmacoEconomics. 355 2015;33(7):673-89.

356 4. Ng M, Fleming T, Robinson M, Thomson B, Graetz N, Margono C, et al. Global, regional, and national prevalence of overweight and obesity in children and adults during 19802013: a systematic analysis for the Global Burden of Disease Study 2013. Lancet (London, England). 2014;384(9945):766-81.

5. Kelly T, Yang W, Chen CS, Reynolds K, He J. Global burden of obesity in 2005 and projections to 2030. International journal of obesity (2005). 2008;32(9):1431-7.

6. Afshin A, Forouzanfar MH, Reitsma MB, Sur P, Estep K, Lee A, et al. Health Effects of Overweight and Obesity in 195 Countries over 25 Years. The New England journal of medicine. 2017;377(1):13-27.

365 7. Hubert HB, Feinleib M, McNamara PM, Castelli WP. Obesity as an independent risk 366 factor for cardiovascular disease: a 26-year follow-up of participants in the Framingham Heart Study. Circulation. 1983;67(5):968-77.
8. Calle EE, Rodriguez C, Walker-Thurmond K, Thun MJ. Overweight, obesity, and mortality from cancer in a prospectively studied cohort of US adults. New England Journal of Medicine. 2003;348(17):1625-38.

9. Molarius A, Seidell J. Selection of anthropometric indicators for classification of abdominal fatness - a critical review. International journal of obesity. 1998;22(8):719.

10. Kragelund C, Omland T. A farewell to body-mass index? The Lancet. 2005;366(9497):1589-91.

11. Sowers JR. Obesity as a cardiovascular risk factor. The American journal of medicine. 2003;115(8):37-41.

12. Tabrizi JS, Sadeghi-Bazargani H, Farahbakhsh M, Nikniaz L, Nikniaz Z. Prevalence and associated factors of prehypertension and hypertension in Iranian Population: The Lifestyle Promotion Project (LPP). PloS one. 2016;11(10):e0165264.

382 14. Larsson SC, Wolk A. Overweight, obesity and risk of liver cancer: a meta-analysis of 383 cohort studies. British journal of cancer. 2007;97(7):1005-8.

384 15. Larsson SC, Wolk A. Obesity and risk of non-Hodgkin's lymphoma: a meta-analysis. 385 International journal of cancer. 2007;121(7):1564-70.

386 16. Larsson SC, Wolk A. Overweight and obesity and incidence of leukemia: a meta-analysis 387 of cohort studies. International journal of cancer. 2008;122(6):1418-21.

388 17. Ohlson L-O, Larsson B, Svärdsudd K, Welin L, Eriksson H, Wilhelmsen L, et al. The 389 influence of body fat distribution on the incidence of diabetes mellitus: 13.5 years of follow-up 390 of the participants in the study of men born in 1913. Diabetes. 1985;34(10):1055-8. 
18. Polednak AP. Estimating the number of U.S. incident cancers attributable to obesity and the impact on temporal trends in incidence rates for obesity-related cancers. Cancer detection and prevention. 2008;32(3):190-9.

394 19. Olsen CM, Green AC, Whiteman DC, Sadeghi S, Kolahdooz F, Webb PM. Obesity and the risk of epithelial ovarian cancer: a systematic review and meta-analysis. European journal of cancer (Oxford, England : 1990). 2007;43(4):690-709. relationship between body weight gain in adulthood and incident type 2 diabetes: a metaanalysis. Obesity reviews : an official journal of the International Association for the Study of Obesity. 2014;15(3):202-14. adolescence on morbidity and premature mortality in adulthood: systematic review. International journal of obesity (2005). 2011;35(7):891-8.

404 22. Narayan KM, Boyle JP, Thompson TJ, Gregg EW, Williamson DF. Effect of BMI on lifetime risk for diabetes in the U.S. Diabetes Care. 2007;30(6):1562-6.

23. Organization WH. Waist circumference and waist-hip ratio: report of a WHO expert consultation, Geneva, 8-11 December 2008. 2011. 24. Sahakyan KR, Somers VK, Rodriguez-Escudero JP, Hodge DO, Carter RE, Sochor O, et al. Normal-Weight Central Obesity: Implications for Total and Cardiovascular Mortality. Annals of internal medicine. 2015;163(11):827-35.

25. Coutinho T, Goel K, Corrêa de Sá D, Carter RE, Hodge DO, Kragelund C, et al. Combining body mass index with measures of central obesity in the assessment of mortality in subjects with coronary disease: role of "normal weight central obesity". Journal of the American College of Cardiology. 2013;61(5):553-60.

26. Eshete T, Kumera G, Bazezew Y, Marie T, Alemu S, Shiferaw K. The coexistence of maternal overweight or obesity and child stunting in low-income country: Further data analysis of the 2016 Ethiopia demographic health survey (EDHS). Scientific African. 2020;9:e00524. 27. Popkin BM, Corvalan C, Grummer-Strawn LM. Dynamics of the double burden of malnutrition and the changing nutrition reality. Lancet (London, England). 2020;395(10217):6574.

28. Boutayeb A. The double burden of communicable and non-communicable diseases in developing countries. Transactions of the Royal Society of Tropical Medicine and Hygiene. 2006;100(3):191-9.

29. Bollyky TJ, Templin T, Cohen M, Dieleman JL. Lower-Income Countries That Face The Most Rapid Shift In Noncommunicable Disease Burden Are Also The Least Prepared. Health affairs (Project Hope). 2017;36(11):1866-75.

\section{Bong C-L, Brasher C, Chikumba E, McDougall R, Mellin-Olsen J, Enright A. The} COVID-19 pandemic: effects on low-and middle-income countries. Anesthesia and analgesia. 2020.

31. Tan MK. COVID-19 in an inequitable world: the last, the lost and the least. Oxford University Press; 2021. p. 493-6.

32. Misganaw A, Haregu TN, Deribe K, Tessema GA, Deribew A, Melaku YA, et al. National mortality burden due to communicable, non-communicable, and other diseases in Ethiopia, 1990-2015: findings from the Global Burden of Disease Study 2015. Population 435 Health Metrics. 2017;15(1):29. 

disability-adjusted life years (DALYs) for 257 diseases and injuries in Ethiopia, 1990-2015: findings from the global burden of disease study 2015. Population Health Metrics. 2017;15(1):28.

440 34. Janakiraman B, Abebe SM, Chala MB, Demissie SF. Central obesity, not BMI explains cardio-metabolic risks among university employees, Ethiopia-a cross-sectional study. 2019. 35. Biru B, Tamiru D, Taye A, Regassa Feyisa B. Central obesity and its predictors among adults in Nekemte town, West Ethiopia. SAGE open medicine. 2021;9:20503121211054988. 36. Dagne S, Menber Y, Petrucka P, Wassihun Y. Prevalence and associated factors of abdominal obesity among the adult population in Woldia town, Northeast Ethiopia, 2020: Community-based cross-sectional study. PloS one. 2021;16(3):e0247960. 37. Derbew Molla M, Fekadu Wolde H. Magnitude of Central Obesity and its Associated Factors Among Adults in Urban Areas of Northwest Ethiopia. 2021. 38. Tesfaye TS, Zeleke TM, Alemu W, Argaw D, Bedane TK. Dietary diversity and physical activity as risk factors of abdominal obesity among adults in Dilla town, Ethiopia. PloS one. 2020;15(7):e0236671.

39. Yazew G, Abate A, Alemseged F, Tewelde T. Factors Associated with Overweight and Central Obesity in Adults at Gilgel Gibe Field Research Center, Southwest Ethiopia: A Secondary Analysis of Data. 2019. surveillance (STEPS) of non-communicable disease risk factors. Global behavioral risk factor surveillance: Springer; 2003. p. 9-22. 41. Organization WH. The WHO STEPwise approach to noncommunicable disease risk factor surveillance. Geneva: World Health Organization. 2005. anesthesiology. 2019;72(6):558. 43. Janakiraman B, Abebe SM, Chala MB, Demissie SF. Epidemiology of general, central obesity and associated cardio-metabolic risks among University Employees, Ethiopia: a crosssectional study. Diabetes, metabolic syndrome and obesity: targets and Therapy. 2020;13:343. 44. Kassie AM, Abate BB, Kassaw MW. Prevalence of overweight/obesity among the adult population in Ethiopia: a systematic review and meta-analysis. BMJ open. 2020;10(8):e039200. 45. Jaacks LM, Vandevijvere S, Pan A, McGowan CJ, Wallace C, Imamura F, et al. The obesity transition: stages of the global epidemic. The lancet Diabetes \& endocrinology. 2019;7(3):231-40. health economics. 2009;28(3):635-48. obesity, abdominal obesity and related risk factors in urban adults from 33 communities of northeast china: the CHPSNE study. BMC Public Health. 2012;12(1):967.

48. Munyogwa MJ, Mtumwa AH. The Prevalence of Abdominal Obesity and Its Correlates among the Adults in Dodoma Region, Tanzania: A Community-Based Cross-Sectional Study. Advances in medicine. 2018;2018:6123156. obesity and its correlates among adults in a peri-urban population of West Africa. AIMS public health. 2019;6(3):334-44. 
50. Zhernakova YV, Zheleznova EA, Chazova IE, Oshchepkova EV, Dolgusheva YA, Yarovaya EB, et al. The prevalence of abdominal obesity and the association with

483 socioeconomic status in Regions of the Russian Federation, the results of the epidemiological

484 study - ESSE-RF. Terapevticheskii arkhiv. 2018;90(10):14-22.

485 51. Henry CJ. Mechanisms of changes in basal metabolism during ageing. European journal 486 of clinical nutrition. 2000;54 Suppl 3:S77-91.

487 52. Tekalegn Y, Engida ZT, Sahiledengle B, Rogers HL, Seyoum K, Woldeyohannes D, et

488 al. Individual and community-level determinants of overweight and obesity among urban men:

489 Further analysis of the Ethiopian demographic and health survey. PloS one.

$490 \quad 2021 ; 16(11): \mathrm{e} 0259412$.

491 53. Chen R, Tunstall-Pedoe H. Socioeconomic deprivation and waist circumference in men 492 and women: The Scottish MONICA surveys 1989 -1995. European Journal of Epidemiology. $493 \quad 2005 ; 20(2): 141-7$.

494 54. Sievert K, Hussain SM, Page MJ, Wang Y, Hughes HJ, Malek M, et al. Effect of 495 breakfast on weight and energy intake: systematic review and meta-analysis of randomised 496 controlled trials. BMJ (Clinical research ed). 2019;364:142.

497 55. Ma X, Chen Q, Pu Y, Guo M, Jiang Z, Huang W, et al. Skipping breakfast is associated 498 with overweight and obesity: A systematic review and meta-analysis. (1871-403X (Print)).

\section{Supporting information}

500 Figure 1: Map of Goba and Robe town, Southeast Ethiopia 

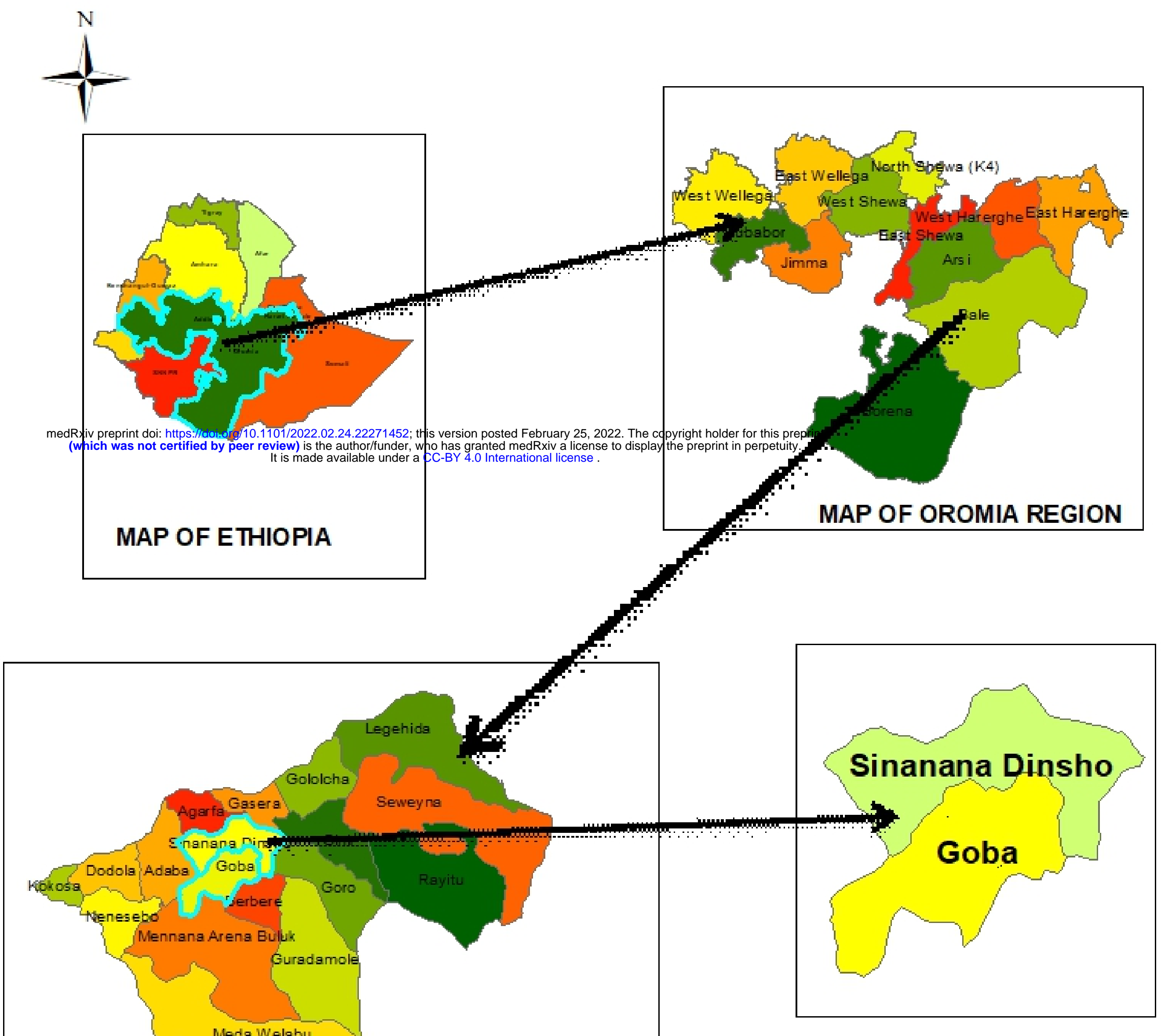

\section{Legend}

MAP OF BALE ZONE

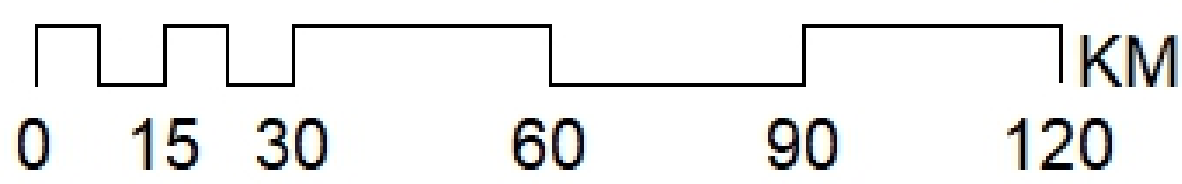

Goba

Sinanana Dinsho 